\title{
COMPARAÇÃO DO EFEITO DO TREINAMENTO PROPRIOCEPTIVO NO TORNOZELO DE NÃO ATLETAS E JOGADORES DE VOLEIBOL
}

\author{
COMPARISON OF THE EFFECT OF PROPRIOCEPTIVE TRAINING ON THE ANKLE OF NON-ATHLETES AND \\ VOLLEYBALL PLAYERS
}

\section{COMPARACIÓN DEL EFECTO DEL ENTRENAMIENTO PROPIOCEPTIVO EN EL TOBILLO DENO ATLETAS Y JUGADORES DE VOLEIBOL}

\author{
Emanuele Lazzari Cristofoli \\ (Fisioterapeuta) \\ Mariana Michalski Peres' \\ (Fisioterapeuta) \\ Lisiane Cecchini² (Fisioterapeuta) \\ Ivan Pacheco ${ }^{2,3}$ \\ (Médico Ortopedista e Traumatologista) \\ Adriana Moré Pacheco \\ (Fisioterapeuta)
}

1. Universidade Federal do Rio Grande do Sul, Porto Alegre, RS, Brasil.

2. Grêmio Náutico União, Porto Alegre, RS, Brasil.

3. Federação Gaúcha de Futebol, Porto Alegre, RS, Brasil.

\section{Correspondência:}

Adriana Moré Pacheco.

Escola de Educação Física,

Fisioterapia e Dança. Universidade Federal do Rio Grande do Sul. Rua: Felizardo, 750, Porto Alegre, RS, Brasil. 90690-200

adrimpacheco@ufrgs.br

\section{RESUMO}

Introdução: O tornozelo é a articulação que sofre mais lesões tanto na vida diária quanto na prática de esportes, especialmente em praticantes de voleibol, que exige movimentos bruscos. Um menor risco de lesão está ligado à estabilidade articular do tornozelo, que pode ser adquirida através de treinamentos proprioceptivos, utilizados para reabilitação e para prevenção de lesões. Objetivo: Avaliar o efeito de um treinamento proprioceptivo para tornozelo por meio do Star Excursion Balance Test (SEBT) em estudantes sedentárias e comparar com resultados obtidos com o mesmo treinamento em atletas do voleibol. Métodos: Onze estudantes sedentárias sem histórico de lesões nos membros inferiores participaram de um treinamento proprioceptivo para os tornozelos, durante quatro semanas. As estudantes foram avaliadas por meio do SEBT antes e após o protocolo de treinamento de propriocepção. Após obtenção das médias pré e pós-teste, foram analisadas intragrupo e comparadas com um banco de dados de estudo que utilizou metodologia idêntica, porém com atletas de voleibol. Resultados: Para a comparação de médias de ambos os tornozelos pré e pós-intervenção das estudantes foi utilizado o teste $t$ pareado. Para comparar os resultados das estudantes com os das atletas foi utilizado o teste $t$ independente. Adotou-se $5 \%(p \leq 0,05)$ como nível de significância e as análises foram realizadas no programa SPSS (Statistical Package for the Social Sciences) versão 18. Os resultados no teste SEBT das estudantes pré e pós-intervenção mostraram diferença significativa em três direções para o tornozelo direito e em quatro direções para o esquerdo. As diferenças entre as estudantes e as atletas foram significativas para duas direções no tornozelo direito e para três no esquerdo. Conclusão: Treinar a propriocepção é eficaz para aumentar a estabilidade tanto de sedentários quanto de atletas, indicando ser um importante meio de prevenção a futuras lesões.

Descritores: avaliação; propriocepção; tornozelo.

\section{ABSTRACT}

Introduction: The ankle is the most frequently injured joint both in daily life and in sports, especially in volleyball players that require sudden movements of the joint. A lower risk of injury is connected to the ankle joint stability, which can be acquired by proprioceptive training, used for rehabilitation and prevention of injuries. Objective: To evaluate the effect of a proprioceptive training for the ankle joint through the Star Excursion Balance Test (SEBT) in sedentary students and compare results with those obtained with the same training in volleyball athletes. Methods: Eleven sedentary students with no history of lower limb injuries participated in a proprioceptive training to the ankles during four weeks. The students were evaluated using the SEBT before and after the protocol of proprioceptive training. After obtaining the pre and post-test averages, they were analyzed intragroup and compared with a database study using the same methodology, but with volleyball players. Results: The paired t test was used to compare the means of both ankles pre and post intervention in students. To compare the outcomes of students with those of the athletes we used the independent $t$ test. We adopted the level of significance $5 \%(p \leq 0.05)$, and analyzes were performed with SPSS (Statistical Package for Social Sciences) version 18. The results of the SEBT testing of the students, pre and post intervention, showed significant differences in three directions for the right ankle and four directions for the left. The differences between students and the athletes were significant for two directions on the right ankle and for three directions on the left. Conclusion: The proprioceptive training is effective to increase the stability of both sedentary students as athletes, indicating that can be an important means to prevent future injuries.

Keywords: evaluation; proprioception; ankle.

\section{RESUMEN}

Introducción: El tobillo es la articulación que sufre más lesiones, tanto en la vida diaria y en el deporte, en especial en los jugadores de voleibol, que requiere movimientos bruscos. Un menor riesgo de lesión está conectado a la estabilidad de la articulación del tobillo, que puede ser adquirida por el entrenamiento propioceptivo utilizado para la 
rehabilitación y para la prevención de lesiones. Objetivo: Evaluar el efecto de un entrenamiento propioceptivo del tobillo a través de la prueba Star Excursion Balance Test (SEBT) en estudiantes sedentarias y comparar con los resultados del mismo entrenamiento en atletas de voleibol. Métodos: Once estudiantes sedentarias, sin antecedentes de lesiones en las extremidades inferiores, participaron en un entrenamiento propioceptivo de los tobillos durante cuatro semanas. Las estudiantes fueron evaluadas por la prueba SEBT antes y después del protocolo de entrenamiento propioceptivo. Después de obtener los promedios de pre y post-test, se analizaron estos valores y se compararon intragrupo y con una base de datos de estudio que utilizó la misma metodología, pero con jugadores de voleibol. Resultados: Para la comparación de promedios de ambos tobillos de las estudiantes antes y después de la intervención se utilizó la pruebat pareada. Para comparar los resultados de las estudiantes con los atletas se utilizó la prueba tindependiente. Se adoptó el $5 \%$ ( $p \leq 0,05)$ como nivel de significación y los análisis se realizaron con el programa SPSS (Statistical Package for the Social Sciences) versión 18. Los resultados de la prueba SEBT de las estudiantes antes y después de la intervención mostraron diferencias significativas en tres direcciones en el tobillo derecho y en cuatro direcciones en el izquierdo. Las diferencias entre las estudiantes y los atletas fueron significativas para ambas direcciones en el tobillo derecho y para tres en el izquierdo. Conclusión: El entrenamiento de propiocepción es eficaz para aumentar la estabilidad tanto de sedentarios como de atletas, lo que indica que es un medio importante para la prevención de lesiones en el futuro.

Descriptores: evaluación; propiocepción; tobillo.

\section{INTRODUÇÃO}

O tornozelo é a articulação que mais frequentemente sofre lesões na vida diária e nas atividades esportivas ${ }^{1,2}$ sendo a entorse a lesão mais comumente observada-5 e o mecanismo de inversão responsável por $80 \%$ a $90 \%$ dos $\operatorname{casos}^{1,2,5,6}$. Dentro das atividades esportivas, o voleibol se destaca como o de maior intercorrência desta lesão, por ser um esporte de contato e utilizar grande quantidade de deslocamentos, saltos e movimentos repentinos ${ }^{2,3,5,7}$. As entorses não se restringem somente as atividades esportivas, podendo ocorrer durante descida de escadas, queda em superfícies irregulares e girando o corpo para o lado oposto ao tornozelo.

Um menor risco de lesão é proporcional à estabilidade do tornozelo, diretamente ligada com a propriocepção, que é um mecanismo de percepção corporal que mantém o controle postural a partir dos estímulos aferentes recebidos sobre movimento e posição ${ }^{8}$. Quando há alterações na propriocepção existe risco para quedas e instabilidade articular ${ }^{9,10}$ além de interferência no controle motor e desempenho funcional da articulação $2,3,5,11$

Treinar a propriocepção aparece como uma alternativa de grande valor na prevenção ou na reabilitação de lesão ligamentar do tornozelo. Esses efeitos positivos podem estar ligados a adaptações neurais centrais e periféricas, maior resistência e flexibilidade ${ }^{12}$. Alguns autores $^{2,12-14}$ demonstraram que os treinos de equilíbrio, com protocolos de exercícios proprioceptivos, para os membros inferiores são capazes de trazer significativas melhorias no desempenho do equilíbrio dinâmico e estes treinamentos têm sido avaliados pelo Star Excursion Balance Test (SEBT), um teste que objetiva medir o nível de estabilidade do tornozelo quando o indivíduo mantém o apoio unipodal do membro a ser avaliado e com o membro contralateral busca-se o máximo de distância que ele consegue atingir em determinadas direções propostas pelo teste 2,12-14.

Como cita Verhagen ${ }^{15}$, sabe-se que metade das entorses recorrentes podem ser prevenidas, porém ainda falta uma visão fundamental para o uso de mecanismos preventivos, quando se trata de tornozelos que nunca sofreram lesões. A literatura é ainda escassa a respeito dos efeitos de um treinamento proprioceptivo sobre tornozelos sem lesões, acarretando a necessidade de investigar o que acontece quando essas articulações estáveis são tratadas.

De acordo com Belangero et al. ${ }^{16}$, estima-se que ocorra uma entorse lateral do tornozelo a cada 10.000 pessoas por dia. Indivíduos que praticam esportes expõem-se a alterações do pH (acidose metabólica), da temperatura, do fluxo sanguíneo, a perda da homeostasia do cálcio, a lesão e fadiga muscular e o acúmulo de produtos do metabolismo celular. A literatura mostra esses fatores como atenuantes do controle neuromuscular, colocando atletas em maior risco de lesão em relação à população em geral ${ }^{11}$.

Tendo em vista a alta incidência de entorse de tornozelo na população e os elementos que podem diminuir o controle neuromuscular em quem pratica esportes, a comparação do efeito de um treinamento proprioceptivo para a articulação do tornozelo entre atletas e não atletas pode ser uma proposta interessante como apresentação de resultados para uma eficácia no tratamento ou prevenção dessa lesão. Portanto, o objetivo desse estudo foi avaliar o efeito de um treinamento proprioceptivo na estabilidade articular do tornozelo pelo teste Star Excursion Balance Test (SEBT) em estudantes sedentárias e comparar com resultados obtidos para o mesmo treinamento em atletas do voleibol.

\section{MATERIAIS E MÉTODOS}

O estudo de Rasool e George ${ }^{12}$ embasou o cálculo amostral (calculado com o programa WinPepi, versão 4.0 (Salt Lake City, Utah, EUA) do estudo, considerando 80\% de confiabilidade e o nível de significância fixado em $5 \%(p \leq 0,05)$. O desvio padrão foi $5 \mathrm{~cm}$ no Star Excursion Balance Test (SEBT), sendo a diferença mínima a ser detectada de 5 cm; assim determinou-se que o tamanho mínimo da amostra de 17 sedentárias com tornozelo estável.

A pesquisa realizada é de caráter quantitativo do tipo semi-experimental onde foram selecionadas, por meio de cartazes informativos da pesquisa, estudantes voluntárias de uma Universidade da cidade de Porto Alegre, RS. Todas eram do gênero feminino, com faixa etária compreendida entre 17 a 22 anos de idade (média 20,45 $\pm 2,115$ ) e índice de massa corporal médio entre 19,56 e 26,84 (média 21,92 $\pm 2,1148$ ). As voluntárias recrutadas não eram atletas, não realizavam treinamento muscular para membros inferiores, nunca participaram de treinos proprioceptivos específicos para tornozelo, não possuíam hiperlassidão ligamentar, avaliadas pelo teste de gaveta anterior do tornozelo e apresentavam as articulações dos tornozelos, joelhos e quadris sem histórico de lesões.

Assim que concordavam em participar da pesquisa, as estudantes assinavam o Termo de Consentimento Livre e Esclarecido e a pesquisa foi aprovada pelo Comitê de Ética em Pesquisa da Universidade Federal do Rio Grande do Sul (UFRGS), em cinco de junho de 2014 sob o número CAAE 27699414.8.0000.5347 e do parecer 675.860, concordando com a Resolução 196/96 do Conselho Nacional de Saúde. 


\section{Procedimentos de coleta dos dados}

As coletas ocorreram, na sala de cinesioterapia, da Clínica de Fisioterapia da Escola de Educação Física, Fisioterapia e Dança da UFRGS, sob supervisão direta da pesquisadora. Foi aplicado um questionário sob a forma de entrevista entre a pesquisadora e a participante na sala de avaliação da Clínica de Fisioterapia com perguntas objetivando a composição da amostra de acordo com os critérios de inclusão e caracterização da amostra final para resultados. Cada participante respondeu individualmente o questionário.

No mesmo dia as estudantes iniciaram a avaliação pré-intervenção no teste proposto para o estudo: o Star Excursion Balance Test. Esse teste consistiu em uma série de mini agachamentos realizados a partir de um posicionamento das estudantes em apoio unipodal no centro de uma rosa dos ventos com oito direções diferentes com ângulos de $45^{\circ}$ entre si. A perna que não estava apoiada tentava alcançar a maior distância da perna apoiada em cada uma das oito direções e, quanto maior a distância atingida, maior a demanda sobre os sistemas de equilíbrio e controle neuromuscular, representando uma maior estabilidade no tornozelo $0^{2,17}$. A mensuração ocorreu três vezes em ambos os tornozelos, sendo registrada a média entre os centímetros atingidos. O teste foi aplicado em ambos os tornozelos. Para tanto, foi utilizado um painel adquirido do estudo de Peres et al.2., que foi posicionado no chão com a proposta do SEBT (Figura 1). 0 posicionamento e a execução de cada avaliação no SEBT foram iguais ao proposto no estudo de Peres et al. ${ }^{2}$ Segundo, Peres et al. ${ }^{2}$ esse teste permite, a mensuração de variáveis de equilíbrio com inferências a propriocepção.

Após o pré-teste, cada estudante participou, três vezes por semana, durante quatro semanas, de um treinamento proprioceptivo baseado no estudo de Hupperets et al. ${ }^{18}$. Esse treinamento consistia em um protocolo de exercícios de propriocepção composto por seis exercícios que evoluíam no grau de exigência sendo realizados com olhos vendados e sobre superfície instável. Ao final dos doze encontros, as participantes eram reavaliadas com o SEBT para ter suas medidas comparadas com o pré-teste.

Ao final das coletas das estudantes os valores obtidos no pré e no pós-teste do SEBT foram analisados e comparados entre si em ambos os tornozelos. Após a comparação intragrupo, os dados passaram por uma comparação intergrupos, na qual foram comparados os resultados da proposta de treinamento proprioceptivo das estudantes sedentárias

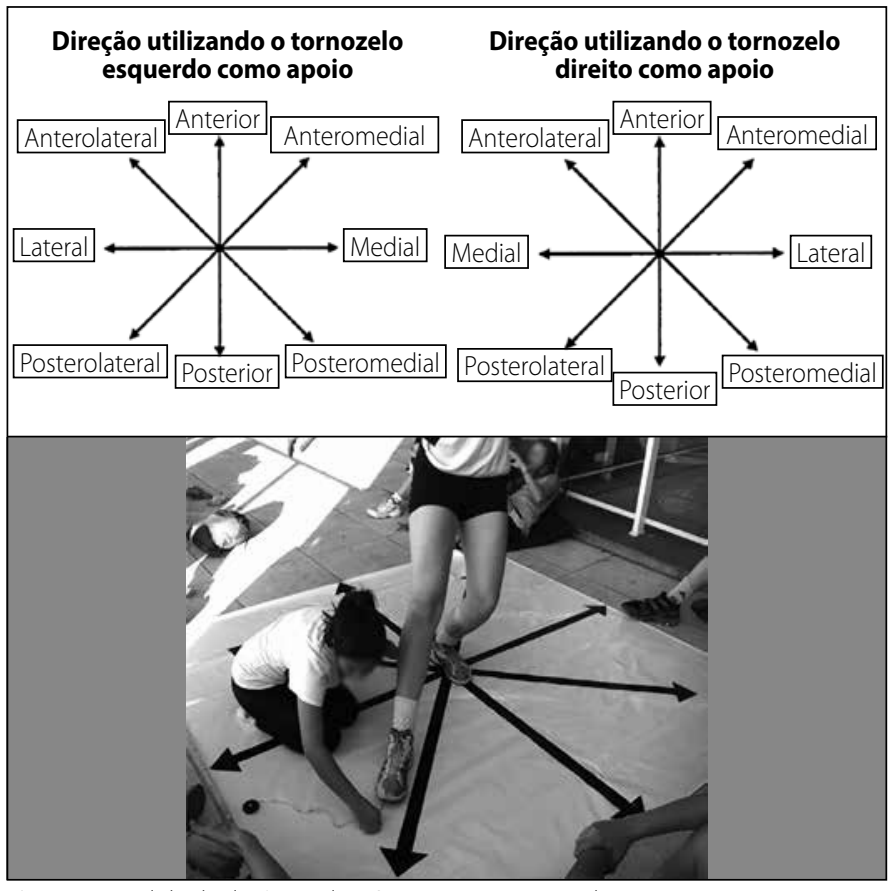

Figura 1. Medida da distância do pé no Star Excursion Balance Test. com atletas femininas de voleibol de mesma faixa etária. Os resultados do treinamento proprioceptivo das atletas de voleibol foram extraídos de um banco de dados do estudo de Peres et al. ${ }^{2}$, que utilizaram uma metodologia idêntica a proposta desta pesquisa.

\section{Análise estatística}

As variáveis quantitativas foram descritas por média e desvio padrão. Para a comparação de pré e pós-intervenção intragrupo das sedentárias utilizou-se o teste $t$ pareado. A fim de comparar o desempenho das sedentárias em relação às atletas, fez-se uma subtração do valor obtido no pós-teste pelo obtido no pré de cada voluntária; dessa forma obteve-se 11 valores de diferença para cada grupo estudado. Em seguida os valores foram submetidos ao teste $t$ para amostras independentes. Ambos os testes tiveram nível de significância fixado em $5 \%(p \leq 0,05)$ e as análises foram realizadas no programa SPSS (Statistical Package for the Social Sciences) versão 18 (Universidade de Chicago, EUA).

\section{RESULTADOS}

Treze estudantes atenderam aos critérios de inclusão do estudo, porém duas abandonaram a pesquisa, restando 11 estudantes avaliadas.

Após o treinamento proprioceptivo proposto, foi comparado o desempenho dos tornozelos direito e esquerdo das estudantes sedentárias no pré e pós- intervenção pelo SEBT teste, onde os resultados mostraram, dentre as oito direções propostas no SEBT, significância estatística na diferença em três direções (posterolateral, póstero medial e antero medial) do tornozelo direito, e em quatro direções (posteromedial, posterolateral, lateral e anteromedial) para o tornozelo esquerdo (Tabelas 1 e 2).

Também foi realizada a comparação das médias do SEBT, coletadas no grupo de estudantes sedentárias com as médias do SEBT de um banco de dados do estudo de Peres et al. ${ }^{2}$, que coletou no grupo de atletas de voleibol. Na comparação do pré e pós-teste entre o grupo das estudantes sedentárias e o grupo das atletas de voleibol encontrou-se diferenças significativas em duas direções (anterior e posterior) para o tornozelo direito, das oito direções avaliadas, e em três direções (medial, posteromedial e lateral) para o tornozelo esquerdo, das oito direções avaliadas (Tabelas 3 e 4).

Tabela 1. Resultados do SEBT pré e pós-intervenção para o tornozelo direito.

\begin{tabular}{c|c|c|c}
\hline Direção & Valor pré $(\mathbf{c m}) \mid \mathbf{D P}$ & Valor pós $(\mathbf{c m}) \mid \mathbf{D P}$ & $\boldsymbol{p}$ \\
\hline Anterior & $54,48 \pm 5,1$ & $54,82 \pm 8,63$ & 0,852 \\
\hline Anterolateral & $45,54 \pm 10,61$ & $46,48 \pm 7,15$ & 0,693 \\
\hline Lateral & $40,51 \pm 12,36$ & $43,78 \pm 11,79$ & 0,186 \\
\hline Posterolateral & $61,84 \pm 10,30$ & $67,02 \pm 7,94$ & $0,039^{*}$ \\
\hline Posterior & $76,14 \pm 9,5$ & $75,99 \pm 6,63$ & 0,928 \\
\hline POsteromedial & $66,66 \pm 10,93$ & $74,99 \pm 8,26$ & $0,003^{*}$ \\
\hline Medial & $55,57 \pm 16,9$ & $62,54 \pm 14,47$ & 0,127 \\
\hline Anteromedial & $47,03 \pm 10,25$ & $54,57 \pm 9,03$ & $0,037^{*}$ \\
\hline${ }^{*}$ p 50,05
\end{tabular}

"p $\leq 0,05$.

Tabela 2. Resultados do SEBT pré e pós-intervenção para o tornozelo esquerdo.

\begin{tabular}{c|c|c|c}
\hline Direção & Valor pré $(\mathbf{c m}) \mid \mathbf{D P}$ & Valor pós $(\mathbf{c m}) \mid \mathbf{D P}$ & $\mathbf{p}$ \\
\hline Anterior & $56,79 \pm 4,48$ & $55,42 \pm 7,72$ & 0,405 \\
\hline Anteromedial & $44,12 \pm 9,53$ & $44,72 \pm 7,43$ & 0,805 \\
\hline Medial & $47,21 \pm 15,33$ & $42,85 \pm 13,97$ & 0,179 \\
\hline Posteromedial & $59,24 \pm 13,24$ & $71,42 \pm 7,64$ & $0,002^{*}$ \\
\hline Posterior & $74 \pm 8,29$ & $73,81 \pm 7,72$ & 0,917 \\
\hline Posterolateral & $61,33 \pm 13,06$ & $71,36 \pm 8,65$ & $0,005^{*}$ \\
\hline Lateral & $51,48 \pm 17,98$ & $67,23 \pm 15,03$ & $0,005^{*}$ \\
\hline Anterolateral & $51,48 \pm 9,72$ & $58,08 \pm 11,48$ & $0,010^{*}$ \\
\hline${ }^{*} \mathbf{p} \leq 0,05$ & &
\end{tabular}


Tabela 3. Comparação entre a diferença de pós e pré-teste média para estudantes e atletas de voleibol (tornozelo direito).

\begin{tabular}{c|c|c|c}
\hline Direção & Diferença estudantes | DP & Diferença atletas | DP & $\mathbf{p}$ \\
\hline Anterior & $0,33 \pm 5,79$ & $6,24 \pm 7,189$ & $0,047^{*}$ \\
\hline Anterolateral & $0,94 \pm 7,66$ & $3,06 \pm 7,52$ & 0,520 \\
\hline Lateral & $3,27 \pm 7,63$ & $5,30 \pm 10,16$ & 0,602 \\
\hline Posterolateral & $5,18 \pm 7,22$ & $6,18 \pm 5,38$ & 0,717 \\
\hline Posterior & $-0,15 \pm 5,39$ & $5,06 \pm 5,16$ & $0,031^{*}$ \\
\hline Posteromedial & $8,33 \pm 7,24$ & $5,60 \pm 6,83$ & 0,375 \\
\hline Medial & $6,97 \pm 13,9$ & $7,14 \pm 7,43$ & 0,970 \\
\hline Anteromedial & $7,54 \pm 10,38$ & $4,11 \pm 6,07$ & 0,356 \\
\hline${ }^{*} p \leq 0,05$. &
\end{tabular}

Tabela 4. Comparação entre a diferença de pós e pré-teste média para estudantes e atletas (tornozelo esquerdo).

\begin{tabular}{l|l|l|l}
\hline Direção & Diferença estudantes $\mid$ DP & Diferença atletas | DP & $\mathbf{p}$ \\
\hline Anterior & $-1,36 \pm 5,19$ & $2,54 \pm 3,74$ & 0,057 \\
\hline Anteromedial & $0,60 \pm 7,94$ & $2,15 \pm 5,44$ & 0,600 \\
\hline Medial & $-4,36 \pm 9,99$ & $6 \pm 4,99$ & $0,006^{*}$ \\
\hline Posteromedial & $12,18 \pm 9,58$ & $4,57 \pm 5,108$ & $0,031^{*}$ \\
\hline Posterior & $-0,18 \pm 5,62$ & $1,27 \pm 5,33$ & 0,541 \\
\hline Posterolateral & $10,02 \pm 9,26$ & $4,48 \pm 4,99$ & 0,096 \\
\hline Lateral & $15,75 \pm 14,64$ & $4,12 \pm 5,59$ & $0,023^{*}$ \\
\hline Anterolateral & $6,60 \pm 6,93$ & $3,85 \pm 8,28$ & 0,408 \\
\hline${ }^{*} \mathrm{p} \leq 0,05$. & &
\end{tabular}

\section{DISCUSSÃO}

Este estudo buscou verificar se um programa de treinamento proprioceptivo tem efeito sobre a estabilidade articular do tornozelo de estudantes sedentárias e se esse efeito age de forma semelhante a um grupo de atletas de voleibol.

Segundo Westlake et al. ${ }^{10}$, os exercícios proprioceptivos demonstram uma grande ação profilática e de reabilitação em lesões musculoesqueléticas. A propriocepção descreve uma variação do tato somada às sensações de posição, movimento articular (cinestesia), por receptores cutâneos de planta do pé, cápsulas articulares e ligamentos ${ }^{19}$. A estimulação constante gera adaptação dos receptores sensoriais, uma das mais importantes bases neurais para adaptação perceptual, o que ajuda a explicar os ganhos proporcionados pelo treinamento proprioceptivo ${ }^{2,4,8,18,20,21}$. Um fator que pode explicar o incremento na estabilidade, tanto em atletas quanto em quem não pratica atividades físicas, é que exercícios proprioceptivos estimulam a atividade dos músculos do tornozelo. Conforme mostrado no estudo de Braun Ferreira et al. ${ }^{22}$, em que 25 atletas sem lesão melhoraram a ativação dos músculos tibiais, fibular longo e gastrocnêmios após exercícios em superfícies instáveis. Da mesma forma, o trabalho de Oliveira et al ${ }^{23}$ treinaram dez indivíduos saudáveis e fisicamente ativos utilizando disco de Freeman e balancim, a fim de avaliar a ativação de reto femoral e tibial anterior. Ao fim do estudo, houve melhora da ativação dos dois grupos musculares, sendo mais significante o aumento em tibial anterior, o que mostra que os músculos do tornozelo podem ser mais importantes para manutenção do equilíbrio do que grandes músculos do membro inferior.

Seguindo a linha de pesquisa da ativação muscular em diferentes superfícies, Cimadoro et al. ${ }^{24}$ avaliaram 13 praticantes de futebol, rugby e handebol com articulação do tornozelo estável. Cada atleta permanecia em apoio unipodal durante cinco segundos em três diferentes pranchas de propriocepção. Os resultados mostraram grande ativação dos músculos tibial anterior, fibular longo, sóleo e extensor longo dos dedos, apontando que o treinamento proprioceptivo pode ser empregado tanto na reabilitação sensório-motora do tornozelo quanto na prevenção de lesões atléticas e prevenção de quedas em adultos e idosos.

Com base nesses conhecimentos, é de suma importância programar intervenções com treinamentos proprioceptivos, sejam elas em articulações com ou sem lesões, que envolvam força e equilíbrio estático e dinâmico para se conseguir resultados como o incremento da estabilidade articular $^{2}$ ou melhora da instabilidade articular.

Assim, o estudo de Rasool e George ${ }^{12}$, selecionaram 16 atletas sem lesão para participaram de um treinamento proprioceptivo de quatro semanas. Após avaliação com o SEBT na segunda e na quarta semana, os atletas treinados mostraram melhora significativa no pré e pós-teste e em relação ao grupo controle. Também, Peres et al. ${ }^{2}$, avaliaram 11 atletas de voleibol feminino sem lesão no tornozelo que participaram de um protocolo com seis exercícios proprioceptivos. Os treinamentos ocorreram três vezes na semana ao longo de um mês e após avaliação no SEBT teste, os resultados mostraram melhora significativa na estabilidade articular do tornozelo.

Além da avaliação de atletas sem lesão, estudos como o de Hupperets et al. ${ }^{18}$ avaliaram a eficácia de um treinamento na diminuição de recorrências de entorses de tornozelo em 256 atletas com entorses recidivantes. Estes realizaram exercícios proprioceptivos três vezes por semana, durante oito semanas. Após um ano os resultados mostraram que o programa de treinamento proprioceptivo foi eficaz apresentando uma diminuição em 35\% das chances de recidivas da lesão. Além desse, Verhagen et al. ${ }^{4}$ utilizaram um protocolo que abrangeu atletas de voleibol de 116 times e obtiveram resultados significativos em relação a diminuição da entorse de tornozelo no grupo intervenção.

Assim como no presente estudo, outras pesquisas também tiveram a intenção de avaliar a proposta de eficácia de um treinamento proprioceptivo em indivíduos não atletas. É o caso do estudo de Braga et al. ${ }^{21}$ que propuseram um treinamento proprioceptivo de dez sessões para uma população de estudantes femininas saudáveis de um curso de fisioterapia. Ao final do estudo houve melhora significativa apontada pelo SEBT mostrando que um treinamento proprioceptivo é eficaz na melhora do equilíbrio corporal.

Outra pesquisa como de Emery et al..$^{25}$ recrutaram 114 estudantes saudáveis de ensino médio, de ambos os sexos, para realizar exercícios com prancha de equilíbrio diariamente durante seis semanas, além de um treino semanal nos seis meses que se sucederam. Ao fim do estudo os estudantes treinados incrementaram sua estabilidade em relação ao grupo controle. O aumento da estabilidade de um grupo que realizou treinamento em relação a um grupo controle também apareceu.

Além da intenção da literatura em mostrar que programas de treinamento proprioceptivos são eficazes tanto para o incremento da estabilidade, quanto para a correção da instabilidade articular, principalmente em tornozelo, vários estudos mostram que não há diferença da utilização desses programas em populações específicas. Nas propostas tanto para sujeitos sem lesão, para atletas sem lesão e também para atletas com histórico de lesão prévia, os treinos de propriocepção apresentaram resultados satisfatórios em seus desempenhos.

Importante ressaltar que mesmo com resultados significativos para a eficácia da propriocepção não foi encontrado na literatura um consenso em relação ao tempo de treinamento e frequência semanal, pois cada protocolo apresenta uma proposta diferente, entretanto sempre relacionando exercícios que envolvem força e treino de equilíbrio das mais diversas formas e com propostas de evoluções graduais².

A outra proposta de discussão relevante é buscar entender a confiabilidade de aprovação da eficácia do treinamento proprioceptivo 
quando proposto em um mesmo teste de avaliação. Muitos estudos têm apontado para a utilização do SEBT como um teste seguro e confiável para a avaliação do incremento de um programa de propriocepção para avaliar a estabilidade ou a instabilidade articular do tornozelo 2,14,21.

Sendo assim, com relação ao SEBT, Hertel et al. ${ }^{14}$ avaliaram o desempenho de 87 indivíduos nas oito direções do teste, firmando ao fim do estudo as direções anteromedial, medial e posteromedial como as mais relevantes para avaliar as condições de estabilidade do tornozelo. Isso corrobora com os achados do presente estudo, que encontrou diferença significativa nas direções anteromedial e posteromedial das estudantes sedentárias e nas direções medial e posteromedial quando se compara o treinamento dessas com o banco de dados das atletas de voleibol do estudo de Peres et al².

As diferenças significativas de pré e pós teste encontradas nas direções supracitadas mostram que o estudo apresentou relevância quanto ao aumento da estabilidade articular do tornozelo em estudantes sedentárias. A semelhança encontrada no efeito do treinamento quando se comparou o grupo de estudantes com o grupo de atletas de voleibol aponta que é possível aumentar a estabilidade do tornozelo realizando um treinamento proprioceptivo tanto em atletas do voleibol quanto em quem não pratica atividades físicas regularmente, consequentemente diminuindo as chances de desenvolver uma lesão.

\section{CONCLUSÃO}

A partir do estudo realizado pôde-se observar que um treinamento proprioceptivo de quatro semanas é uma alternativa viável e eficaz no incremento da estabilidade articular do tornozelo, tanto em atletas de voleibol quanto em indivíduos sedentários. A efetividade do treino de propriocepção mesmo em pessoas que não realizam treinamento muscular pode ser explicada pela grande ativação de músculos estabilizadores dos membros inferiores que ocorre durante posturas que demandam equilíbrio do indivíduo. Para esta pesquisa, assim como na literatura, a variação metodológica, o tamanho amostral, os cálculos estatísticos e diferentes populações são fatores que podem interferir em muitos resultados.

Todos os autores declararam não haver qualquer potencial conflito de interesses referente a este artigo.

CONTRIBUIÇÕES DOS AUTORES: Cada autor contribuiu individual e significativamente para o desenvolvimento do manuscrito. ELC (0000-0001-5438-9267)* e AMP (0000-0001-9781-1413)* foram os principais contribuintes na redação do manuscrito. MMP (0000-0001-9029-9541)* e LC (0000-0002-6855-2616)* contribuíram na primeira parte da coleta de dados com as atletas de voleibol. ELC realizou a coleta de dados da segunda parte do estudo e a pesquisa bibliográfica. ELC, AMP e IP (0000-0002-3109-3866)* contribuíram com a concepção intelectual do estudo e discutiram os resultados e revisão final do manuscrito. AMP e IP* revisaram o artigo para a publicação. Todos os autores contribuíram com o conceito intelectual do estudo. *ORCID (Open Researcher and Contributor ID).

\section{REFERÊNCIAS}

1. Silva RS, Silva AP, Sônego DA, Paula NM. Alterações neuromusculares no quadril associadas a entorses do tornozelo: revisão de literatura. Fisioter Mov. 2011;24(3):503-11.

2. Peres MM, Cecchini L, Pacheco I, Pacheco AM. Efeitos do treinamento proprioceptivo na estabilidade do tornozelo em atletas de voleibol. Rev Bras Med Esporte. 2014;20(2):146-50.

3. Meurer MC, Pacheco AM, Pacheco I, Silva MF. Análise da influência da bandagem funcional de tornozelo no tempo de reação do fibular longo em sujeitos saudáveis. Rev Bras Med Esporte. 2010;16(3):196-200.

4. Verhagen E, van der Beek A, Twisk J, Bouter L, Bahr R, van Mechelen W. The effect of a proprioceptive balance board-training program for the prevention of ankle sprains: a prospective controlled trial. Am J Sports Med. 2004;32(6):1385-93.

5. Pacheco AM, Vaz MA, Pacheco I. Avaliação do tempo de resposta eletromiográfica em atletas de voleibol e não atletas que sofreram entorse de tornozelo. Rev Bras Med Esporte. 2005;11(6):325-30.

6. Junge A, Langevoort G, Pipe A, Peytavin A, Wong F, Mountjoy M. Injuries in team sport tournaments during the 2004 Olympic Games. Am J Sports Med. 2006;34(4):565-76.

7. Almeron MM, Pacheco AM, Pacheco I. Relação entre fatores de risco intrínsecos e extrínsecos e a prevalência de lesões em membros inferiores em atletas de basquetebol e voleibol. Ciência \& Saúde. 2009;2(2):58-65.

8. Baldaço FO, Cadó VP, Souza J, Mota CB, Lemos JC. Análise do treinamento proprioceptivo no equilíbrio de atletas de futsal feminino. Fisioter Mov. 2010;23(2): 183-92

9. Prado MP;Fernandes TD, Camanho GL, Mendes AAM, Amodio DT. Mechanical instability after acute ankle ligament injury: randomized prospective comparison of two forms of conservative treatment. Rev Bras Ortop. 2013;48(4):307-16.

10. Westlake KP, Wu Y, Culham EG. Sensory-specific balance training in older adults: effect on position, movement, and velocity sense at the ankle. Phys Ther. 2007;87(5):560-8

11. Souza RZ, Bastos FN, Vanderlei LCM, Júnior JN, Pastre CM. Atualização sobre modelos de avaliação e treinamento proprioceptivo para articulação do tornozelo. Terapia Manual. 2010;8(40):179-82.

12. Rasool J, George K. The impact of single-leg dynamic balance training on dynamic stability. Phys Ther in Sport. 2007;8(4):177-84.

13. Meneghini T, Rempel C, Barnes DD, Duarte F, Périco E. Avaliação da ativação proprioceptiva em atletas amadoras de voleibol. ConScientiae Saúde. 2009;8(1):47-55.
14. Hertel J, Braham RA, Hale SA, Olmsted-Kramer LC. Simplifying the star excursion balance test: analyses of subject wich and without chronic ankle instability. J Ortop Sports Phys Ther. 2006;36(3):131-7.

15. Verhagen E. How fundamental knowledge aids implementation: ankle sprains as an example. Acta Med Port. 2013;26(2):171-74.

16. Belangero PS, Tamaoki MJS, Nakama GY, Shoiti MV, Gomes RVF, Belloti JC. Como o ortopedista brasileiro trata entorse lateral aguda do tornozelo? Rev Bras Ortop. 2010;45(5):468-73.

17. Earl JE, Hertel J. Lower-extremity muscle activation during the Star Excursion Balance Tests. J Sport Rehabil. 2001;10:93-104.

18. Hupperets MD, Verhagen EA, Van Mechelen W. Effect of unsupervised home based proprioceptive training on recurrences of ankle sprain: randomised controlled trial. BMJ. 2009;339:b2684.

19. Lokhande MV, Shetye J, Mehta A, Deo MV. Assessment of knee joint proprioception in weight bearing and in non-weight bearing positions in normal subjects. JKIMSU. 2013;2(2):94-101.

20. O'Driscoll J, Kerin F, Delahunt E. Effect of a 6-week dynamic neuromuscular training programme on ankle joint function: A case report. Sports Med Arthrosc Rehabil Ther Technol. 2011;3(13);1-7.

21. Braga MM, Nunes GS, Schutz GR, Menezes FS. Treinamento sensório-motor com Nintendo Wii ${ }^{\oplus}$ e disco proprioceptivo: efeitos sobre o equilíbrio de mulheres jovens saudáveis. R bras Ci e Mov. 2012;20(3):37-45

22. Braun Ferreira LA, Pereira WM, Rossi LP, Kerpers II, Paula Jr ARP, Oliveira CS. Analysis of electromyographic activity of ankle muscles on stable and unstable surfaces with eyes open and closed. J Bodyw Mov Ther. 2011;15:496-501

23. Oliveira DCS, Santos PAM, Rezende L, Silva MR, Lizardo FB, Sousa GC, et al. Análise eletromiográfica de músculos do membro inferior em exercícios proprioceptivos realizados com olhos abertos e fechados. Rev Bras Med Esporte. 2012;18:261-6.

24. Cimadoro G, Paizis C, Alberti G, Babault N. Effects of different unstable supports on EMG activity and balance. Neurosci Lett. 2013;548:228-32.

25. Emery CA, Cassidy JD, Klassen TP, Rosychuk RJ, Rowe BH. Effectiveness of a home-based balance-training program in reducing sports-related injuries among healthy adolescents: a cluster randomized controlled trial. CMAJ. 2005;172(6):749-54. 